\title{
Tabelle zur Berechnung von chemischen Analysen
}

\author{
berechnet von
}

W. D. Treadwell.

(Herausgegeben von der Schweizerischen Atomgewichtskommission.)

(3. XI, 21.)

Die folgende Tabelle ist im Auftrag der schweizerischen Atomgewichtskommission aufgestellt worden, unter Verwendung der ron dieser Kommission vorgeschlagenen Atomgewichte.

Mit dem Einverständnis von Herm Prof. Thiel, dem Herausgeber von $F$. W. Küster's ,Logarithmischen Rechentafeln für Chemiker"., haben wir die dort in Tabelle IV gewählte Anordnung übernommen. Für sein Entgegenkommen sprechen wir Herm Prof. Thiel an dieser Stelle unsern verbindlichen Dank aus.

\begin{tabular}{|c|c|c|c|}
\hline $\begin{array}{l}\text { Gesucht } \\
\text { cherehé }\end{array}$ & $\begin{array}{l}\text { Gefunden } \\
\text { trouvé }\end{array}$ & $\begin{array}{l}\text { Faktor } \\
\text { facteur }\end{array}$ & $\begin{array}{l}\text { Log. (Mantissen) } \\
\text { log. (mantisses) }\end{array}$ \\
\hline $\mathrm{Ag}$ & $\begin{array}{l}\mathrm{AgBr} \\
\mathrm{AgCl} \\
\mathrm{Ag}_{2} \mathrm{~S}\end{array}$ & $\begin{array}{l}0,5744 \\
0,7526 \\
0,8706\end{array}$ & $\begin{array}{l}75924 \\
87657 \\
93982\end{array}$ \\
\hline $\mathrm{Al}$ & $\mathrm{Al}_{2} \mathrm{O}_{3}$ & 0,5303 & 72455 \\
\hline As & $\begin{array}{l}\mathrm{As}_{2} \mathrm{~S}_{3} \\
\mathrm{As}_{2} \mathrm{~S}_{5} \\
\left(\mathrm{NH}_{4} \mathrm{MgAsO}_{4}\right)_{2} \cdot \mathrm{H}_{2} \mathrm{O} \\
\mathrm{Mg}_{2} \mathrm{As}_{2} \mathrm{O}_{7} \\
\mathrm{Mg}_{2} \mathrm{P}_{2} \mathrm{O}_{7}\end{array}$ & $\begin{array}{l}0,6091 \\
0,4832 \\
0,3939 \\
0,4827 \\
0.6731\end{array}$ & $\begin{array}{l}78470 \\
68412 \\
59533 \\
68371 \\
89810\end{array}$ \\
\hline $\mathrm{As}_{2} \mathrm{O}_{3}$ & $\begin{array}{l}\mathrm{As}_{2} \mathrm{~S}_{3} \\
\mathrm{As}_{2} \mathrm{~S}_{5} \\
\left(\mathrm{NH}_{4} \mathrm{MgAsO}\right)_{4} \cdot \mathrm{H}_{2} \mathrm{O} \\
\mathrm{Mg}_{2} \mathrm{As}_{2} \mathrm{O}_{7} \\
\mathrm{Mg}_{2} \mathrm{P}_{2} \mathrm{O}_{7}\end{array}$ & $\begin{array}{l}0,8041 \\
0,6379 \\
0,5200 \\
0.6373 \\
0,8880\end{array}$ & $\begin{array}{l}90533 \\
80475 \\
71596 \\
80435 \\
94843\end{array}$ \\
\hline$A s_{2} O_{5}$ & $\begin{array}{l}\mathrm{As}_{s_{2}} \mathrm{~S}_{3} \\
\mathrm{~A}_{s_{2}} \mathrm{~S}_{5}\end{array}$ & $\begin{array}{l}0,9342 \\
0,7410\end{array}$ & $\begin{array}{l}97042 \\
86984\end{array}$ \\
\hline
\end{tabular}


- $1011-$

\begin{tabular}{|c|c|c|c|}
\hline $\begin{array}{l}\text { Gesucht } \\
\text { cherché }\end{array}$ & $\begin{array}{l}\text { Gefunden } \\
\text { trouvé }\end{array}$ & $\begin{array}{l}\text { Faktor } \\
\text { facteur }\end{array}$ & $\begin{array}{l}\text { Log. (Mantissen) } \\
\text { log. (mantisses) }\end{array}$ \\
\hline \multirow{3}{*}{$\mathrm{As}_{2} \mathrm{O}_{5}$} & $\left(\mathrm{NH}_{4} \mathrm{MgAsO}_{4}\right)_{2} \cdot \mathrm{H}_{2} \mathrm{O}$ & 0,6040 & 78104 \\
\hline & $\mathrm{Mg}_{2} \mathrm{As}_{2} \mathrm{O}_{7}$ & 0,7404 & 86944 \\
\hline & $\mathrm{Mg}_{2} \mathrm{P}_{2} \mathrm{O}_{7}$ & 1,0323 & 01382 \\
\hline \multirow{5}{*}{$\mathrm{AsO}_{3}$} & $\mathrm{As}_{2} \mathrm{~S}_{3}$ & 0,9992 & 99964 \\
\hline & $\mathrm{As}_{2} \mathrm{~S}_{5}$ & 0,7925 & 89906 \\
\hline & $\left(\mathrm{NH}_{4} \mathrm{MgAsO}_{4}\right)_{2} \cdot \mathrm{H}_{2} \mathrm{O}$ & 0,6461 & 81027 \\
\hline & $\mathrm{Mg}_{2} \mathrm{As}_{2} \mathrm{O}_{7}$ & 0,7919 & 89866 \\
\hline & $\mathrm{Mg}_{2} \mathrm{P}_{2} \mathrm{O}_{7}$ & 1,1042 & 04304 \\
\hline \multirow[t]{5}{*}{$\mathrm{AsO}_{4}$} & $\mathrm{As}_{2} \mathrm{~S}_{3}$ & 1,1291 & 05276 \\
\hline & $\mathrm{As}_{2} \mathrm{~S}_{5}$ & 0,8958 & 95218 \\
\hline & $\left(\mathrm{NH}_{4} \mathrm{MgAsO}_{4}\right)_{2} \cdot \mathrm{H}_{2} \mathrm{O}$ & 0,7301 & 86339 \\
\hline & $\mathrm{Mg}_{2} \mathrm{As}_{2} \mathrm{O}_{7}$ & 0,8950 & 95178 \\
\hline & $\mathrm{Mg}_{2} \mathrm{P}_{2} \mathrm{O}_{7}$ & 1,2479 & 09616 \\
\hline$B$ & $\overline{\mathrm{B}_{2} \mathrm{O}_{3}}$ & 0,3123 & 49460 \\
\hline $\mathrm{BO}_{3}$ & $\mathrm{~B}_{2} \mathrm{O}_{3}$ & 1,6877 & 22729 \\
\hline $\mathrm{B}_{4} \mathrm{O}_{7}$ & $\mathrm{~B}_{2} \mathrm{O}_{3}$ & 1,1146 & 04712 \\
\hline \multirow[t]{4}{*}{$\mathrm{Ba}$} & $\mathrm{BaCO}_{3}$ & 0,6961 & 84264 \\
\hline & $\mathrm{BaCrO}_{4}$ & 0,5422 & 73418 \\
\hline & $\mathrm{BaSO}_{4}$ & 0,5885 & 76976 \\
\hline & $\mathrm{BaSiF}_{6}$ & 0,4912 & 69130 \\
\hline \multirow[t]{4}{*}{$\mathrm{BaO}$} & $\mathrm{BaCO}_{3}$ & 0,7771 & 89048 \\
\hline & $\mathrm{BaCrO}_{4}$ & 0,6054 & 78202 \\
\hline & $\mathrm{BaSO}_{4}$ & 0,6571 & 81760 \\
\hline & $\mathrm{BaSiF}_{6}$ & 0,5484 & 73914 \\
\hline $\mathrm{Be}$ & $\mathrm{BeO}$ & 0,3625 & 55934 \\
\hline \multirow[t]{4}{*}{$\mathrm{Bi}$} & $\mathrm{Bi}_{2} \mathrm{O}_{3}$ & 0,8965 & 95257 \\
\hline & $\mathrm{BiOCl}$ & 0,8017 & 90399 \\
\hline & $\mathrm{BiPO}_{4}$ & 0,6880 & 83756 \\
\hline & $\mathrm{Bi}_{2} \mathrm{~S}_{3}$ & 0,8122 & 90965 \\
\hline \multirow[t]{4}{*}{$\mathrm{Bi}_{2} \mathrm{O}_{3}$} & $\mathrm{BiOCl}$ & 0,8942 & 95142 \\
\hline & $\mathrm{BiPO}_{4}$ & 0,7656 & 88399 \\
\hline & $\mathrm{Bi}_{2} \mathrm{~S}_{3}$ & 0,9059 & $95 \% 07$ \\
\hline & $\mathrm{Bi}$ & 1,1154 & 04742 \\
\hline \multirow[t]{2}{*}{$\mathrm{Br}$} & $\mathrm{AgBr}$ & 0,4256 & 62896 \\
\hline & $\mathrm{AgCl}$ & 0,5576 & 74629 \\
\hline
\end{tabular}


- 1012

\begin{tabular}{|c|c|c|c|}
\hline $\begin{array}{l}\text { Gesucht } \\
\text { cherché }\end{array}$ & $\begin{array}{c}\text { Gefunden } \\
\text { trouvé }\end{array}$ & $\begin{array}{l}\text { Faktor } \\
\text { facteur }\end{array}$ & $\begin{array}{l}\text { Log. (Mantissen) } \\
\text { log. (mantisses) }\end{array}$ \\
\hline $\begin{array}{l}\mathrm{C} \\
\mathrm{CN} \\
\mathrm{CO}_{3} \\
\mathrm{CO}_{2} \\
\mathrm{CO}_{3} \\
\end{array}$ & $\begin{array}{l}\mathrm{CO}_{2} \\
\mathrm{AgCN} \\
\mathrm{CO}_{2} \\
\mathrm{CaCO}_{3} \\
\mathrm{CaO} \\
\mathrm{CO}_{2} \\
\end{array}$ & $\begin{array}{l}0,2727 \\
0,1943 \\
1,3636 \\
0,4396 \\
0,7844 \\
1,3636 \\
\end{array}$ & $\begin{array}{l}43573 \\
28836 \\
13470 \\
64306 \\
89456 \\
13470 \\
\end{array}$ \\
\hline $\begin{array}{l}\mathrm{CaO} \\
\mathrm{Ca}_{3}\left(\mathrm{PO}_{4}\right)_{2} \\
\mathrm{CaSO}_{4}\end{array}$ & $\begin{array}{l}\mathrm{CaCO}_{3} \\
\mathrm{CaO} \\
\mathrm{CaSO}_{4} \\
\mathrm{CaF}_{2} \\
\mathrm{CaCO}_{3} \\
\mathrm{CaSO}_{4} \\
\mathrm{CaF}_{2} \\
\mathrm{CO}_{2} \\
\mathrm{Mg}_{2} \mathrm{P}_{2} \mathrm{O}_{7} \\
\mathrm{BaSO}_{4}\end{array}$ & $\begin{array}{l}0,4004 \\
0,7147 \\
0,2943 \\
0,51333 \\
0,5603 \\
0,4119 \\
0,7182 \\
1,2713 \\
1,3932 \\
0,5831 \\
\end{array}$ & $\begin{array}{l}60252 \\
85409 \\
46883 \\
71034 \\
74843 \\
61474 \\
85625 \\
10528 \\
14401 \\
765 \% 6\end{array}$ \\
\hline $\begin{array}{l}\text { cd } \\
\text { cao }\end{array}$ & $\begin{array}{l}\mathrm{CdO} \\
\mathrm{CdSO}_{4} \\
\mathrm{Cd} \\
\mathrm{CdSO}_{4}\end{array}$ & $\begin{array}{l}0,8754 \\
0,5892 \\
1,1424 \\
0,6159\end{array}$ & $\begin{array}{l}94280 \\
73172 \\
05780 \\
78952\end{array}$ \\
\hline ('e & $\begin{array}{l}\mathrm{Ce}_{2} \mathrm{O}_{3} \\
\mathrm{CeO}_{2}\end{array}$ & $\begin{array}{l}0,85: 9 \\
0,814: 3\end{array}$ & $\begin{array}{l}93142 \\
9107 \%\end{array}$ \\
\hline $\mathrm{Cl}$ & $\begin{array}{l}\mathrm{Ag} \\
\mathrm{AgCl}\end{array}$ & $\begin{array}{l}0,3287 \\
0,2474\end{array}$ & $\begin{array}{l}.51680 \\
.39333\end{array}$ \\
\hline $\mathrm{CHH}$ & $\begin{array}{l}\mathrm{Ag} \\
\mathrm{AgCl}\end{array}$ & $\begin{array}{l}0,3380 \\
0,2541\end{array}$ & $\begin{array}{l}52897 \\
40554\end{array}$ \\
\hline $\mathrm{ClO}_{3}$ & $\begin{array}{l}\mathrm{AgCl} \\
\mathrm{KCl} \\
\mathrm{NaCl}\end{array}$ & $\begin{array}{l}0,6828 \\
1,1194 \\
1,4276\end{array}$ & $\begin{array}{l}70511 \\
0489 \% \\
1546 \%\end{array}$ \\
\hline$(1)_{3} \mathrm{~K}$ & $\begin{array}{l}\mathrm{AgCl} \\
\mathrm{KCl}\end{array}$ & $\begin{array}{l}0,8550 \\
1,6438\end{array}$ & $\begin{array}{l}93198 \\
21.584\end{array}$ \\
\hline $\begin{array}{l}\left(\mathrm{CO}_{3} \mathrm{Na}\right. \\
\mathrm{ClO}_{4}\end{array}$ & $\begin{array}{l}\mathrm{AgCl} \\
\mathrm{NaCl} \\
\mathrm{AgCl} \\
\mathrm{KCl}\end{array}$ & $\begin{array}{l}0,7427 \\
1,8211 \\
0,69339 \\
1,3340\end{array}$ & $\begin{array}{l}87082 \\
26033 \\
84128 \\
12514\end{array}$ \\
\hline
\end{tabular}


- $1013-$

\begin{tabular}{|c|c|c|c|}
\hline $\begin{array}{l}\text { Gesucht } \\
\text { cherché }\end{array}$ & $\begin{array}{l}\text { Gefunden } \\
\text { trouvé }\end{array}$ & $\begin{array}{l}\text { Faktor } \\
\text { facteur }\end{array}$ & $\begin{array}{l}\text { Log. (Mantissen) } \\
\log \text {. (mantisses) }\end{array}$ \\
\hline \multirow{3}{*}{$\begin{array}{l}\mathrm{ClO}_{4} \\
\mathrm{ClO}_{4} \mathrm{~K}\end{array}$} & $\mathrm{NaCl}$ & 1,7013 & 23079 \\
\hline & $\mathrm{AgCl}$ & 0,9667 & 98527 \\
\hline & $\mathrm{KCl}$ & 1,8584 & 26913 \\
\hline \multirow[t]{2}{*}{$\mathrm{ClO}_{4} \mathrm{Na}$} & $\mathrm{AgCl}$ & 0,8543 & 93163 \\
\hline & $\mathrm{NaCl}$ & 2,0948 & 32114 \\
\hline \multirow[t]{2}{*}{ CN } & $\mathrm{Ag}$ & 0,2411 & 38220 \\
\hline & $\mathrm{AgCN}$ & 0,1943 & 28836 \\
\hline \multirow[t]{3}{*}{ CNS } & $\mathrm{AgCNS}$ & $0,34.99$ & 54399 \\
\hline & $\mathrm{BaSO}_{4}$ & 0,2488 & 39585 \\
\hline & CuCNS & 0,4774 & 67888 \\
\hline \multirow{3}{*}{$\begin{array}{l}\mathrm{Co} \\
\mathrm{CoO}\end{array}$} & $\mathrm{CoSO}_{4}$ & 0,3804 & 58019 \\
\hline & Co & 1,2713 & 10426 \\
\hline & $\mathrm{CoSO}_{4}$ & 0,4836 & 68445 \\
\hline \multirow[t]{3}{*}{$\mathrm{Cr}$} & $\mathrm{BaCrO}_{4}$ & 0,2052 & 31219 \\
\hline & $\mathrm{Cr}_{2} \mathrm{O}_{3}$ & 0,6842 & 83519 \\
\hline & $\mathrm{PbCrO}_{4}$ & 0,1609 & 20653 \\
\hline \multirow[t]{2}{*}{$\mathrm{Cr}_{2} \mathrm{O}_{3}$} & $\mathrm{BaCrO}_{4}$ & 0,2999 & 47700 \\
\hline & $\mathrm{PbCrO}_{4}$ & 0,2352 & 37134 \\
\hline \multirow[t]{3}{*}{$\mathrm{CrO}_{3}$} & $\mathrm{BaCrO}_{4}$ & 0,3947 & 59619 \\
\hline & $\mathrm{Cr}_{2} \mathrm{O}_{3}$ & 1,3160 & 11919 \\
\hline & $\mathrm{PbCrO}_{4}$ & 0,3094 & 49053 \\
\hline \multirow{3}{*}{$\mathrm{CrO}_{4}$} & $\mathrm{BaCrO}_{4}$ & 0,4578 & 66065 \\
\hline & $\mathrm{Cr}_{2} \mathrm{O}_{3}$ & 1,5263 & 18365 \\
\hline & $\mathrm{PbCrO}_{4}$ & 0,3589 & 55499 \\
\hline $\mathrm{Cs}$ & $\mathrm{Cs}_{2} \mathrm{SO}_{4}$ & 0,7340 & 86569 \\
\hline \multirow[t]{3}{*}{$\overline{\mathrm{Cu}}$} & $\mathrm{CuO}$ & 0,7989 & 90250 \\
\hline & CuCNS & 0,5226 & 71814 \\
\hline & $\mathrm{Cu}_{2} \mathrm{~S}$ & 0,7986 & 90231 \\
\hline \multirow[t]{3}{*}{$\mathrm{CuO}$} & $\mathrm{Cu}$ & 1,2517 & 09750 \\
\hline & CuCNS & 0,6541 & 81564 \\
\hline & $\mathrm{Cu}_{2} \mathrm{~S}$ & 0,9996 & 99981 \\
\hline Err & $\mathrm{Er}_{2} \mathrm{O}_{3}$ & $0, \overline{8746}$ & 94182 \\
\hline \multirow[t]{3}{*}{$F$} & $\mathrm{CaF}_{2}$ & 0,4866 & 68719 \\
\hline & $\mathrm{CaSO}_{4}$ & 0,2791 & 44573 \\
\hline & $\mathrm{SiF}_{4}$ & 0,7287 & 86253 \\
\hline
\end{tabular}


- $1014-$

\begin{tabular}{|c|c|c|c|}
\hline $\begin{array}{l}\text { Gesucht } \\
\text { cherché }\end{array}$ & $\begin{array}{l}\text { Gefunden } \\
\text { trouvé }\end{array}$ & $\begin{array}{l}\text { Faktor } \\
\text { facteur }\end{array}$ & $\begin{array}{l}\text { Log. (Mantissen) } \\
\log \text {. (mantisses) }\end{array}$ \\
\hline $\mathrm{Fe}$ & $\mathrm{Fe}_{2} \mathrm{O}_{3}$ & 0,6994 & 84473 \\
\hline \multirow[t]{2}{*}{$\mathrm{FeO}$} & $\mathrm{Fe}$ & 1,2865 & 10942 \\
\hline & $\mathrm{Fe}_{2} \mathrm{O}_{3}$ & 0,8998 & 95145 \\
\hline $\mathrm{Fe}_{2} \mathrm{O}_{3}$ & $\mathrm{Fe}$ & 1,4298 & 15527 \\
\hline $\mathrm{FeS}_{2}$ & $\mathrm{Fe}_{2} \mathrm{O}_{3}$ & 1,5028 & 17689 \\
\hline $\mathrm{H}$. & $\mathrm{H}_{2} \mathrm{O}$ & 0,1119 & 04884 \\
\hline $\mathrm{HBr}$ & $\mathrm{AgBr}$ & 0,430 ! & 63441 \\
\hline $\mathrm{HCl}$ & $\mathrm{AgCl}$ & 0,2544 & 40557 \\
\hline HJ & AgJ & 0,5448 & 73627 \\
\hline \multirow{5}{*}{$\mathrm{HNO}_{3}$} & $\mathrm{C}_{20} \mathrm{H}_{16} \mathrm{~N}_{4} \cdot \mathrm{HNO}_{3}$ & 0,1680 & 22522 \\
\hline & $\mathrm{NH}_{4} \mathrm{Cl}$ & 1,1779 & $0 \% 110$ \\
\hline & $\left(\mathrm{NH}_{4}\right)_{2} \mathrm{PtCl}_{6}$ & 0,2838 & 45306 \\
\hline & NO & 2,1000 & 32221 \\
\hline & $\mathrm{Pt}$ & 0,6457 & 81000 \\
\hline \multirow[t]{2}{*}{$\mathrm{Hg}$} & $\mathrm{HgCl}$ & 0,8495 & 92917 \\
\hline & $\mathrm{HgS}$ & 0,8620 & $9354 \%$ \\
\hline \multirow[t]{4}{*}{$J$} & $\mathrm{Ag}$ & $1,176.5$ & 07059 \\
\hline & $\mathrm{AgCl}$ & 0,8855 & $94 \% 16$ \\
\hline & $\mathrm{AgJ}$ & 0,5405 & 73283 \\
\hline & $\mathrm{PdJ}_{2}$ & 0,7041 & 84763 \\
\hline \multirow[t]{5}{*}{$\mathrm{K}$} & $\mathrm{KCl}$ & 0,5244 & 71967 \\
\hline & $\mathrm{K}_{2} \mathrm{SO}_{4}$ & 0,4487 & 65198 \\
\hline & $\mathrm{KClO}_{4}$ & 0,2822 & 45056 \\
\hline & ${ }^{*} \mathrm{~K}_{2} \mathrm{PtCl}_{6}$ & $0,160: 3$ & 20482 \\
\hline & $\mathrm{Pt}$ & 0,4006 & 60273 \\
\hline \multirow[t]{4}{*}{$\mathrm{KCl}$} & $\mathrm{K}_{2} \mathrm{SO}_{4}$ & 0,8557 & 93231 \\
\hline & $\mathrm{KClO}_{4}$ & 0,5381 & $7308 \%$ \\
\hline & ${ }^{*} \mathrm{~K}_{2} \mathrm{PtCl}_{6}$ & 0,3056 & 48516 \\
\hline & $\mathrm{Pt}$ & 0,7639 & 88306 \\
\hline \multirow[t]{5}{*}{$\mathrm{K}_{2} \mathrm{O}$} & $\mathrm{KCl}$ & 0,6317 & 80051 \\
\hline & $\mathrm{K}_{2} \mathrm{SO}_{4}$ & $0,540.5$ & 73283 \\
\hline & $\mathrm{KClO}_{4}$ & 0,3399 & 53141 \\
\hline & ${ }^{*} \mathrm{~K}_{2} \mathrm{PtCl}_{6}$ & 0,1981 & 28566 \\
\hline & $\mathrm{Pt}$ & 0,4826 & 68357 \\
\hline $\mathrm{K}_{2} \mathrm{SO}_{4}$ & $\mathrm{BaSO}_{4}$ & 0,7465 & 87305 \\
\hline La & $\mathrm{La}_{2} \mathrm{O}_{3}$ & $0,8 . \overline{27}$ & 93082 \\
\hline
\end{tabular}

*) empirisch. 


\begin{tabular}{|c|c|c|c|}
\hline $\begin{array}{l}\text { Gesucht } \\
\text { cherché }\end{array}$ & $\begin{array}{l}\text { Gefunden } \\
\text { trouvé }\end{array}$ & $\begin{array}{l}\text { Faktor } \\
\text { facteur }\end{array}$ & $\begin{array}{l}\text { Log. (Mantissen) } \\
\text { log. (mantisses) }\end{array}$ \\
\hline \multirow[t]{2}{*}{$\mathrm{Li}$} & $\mathrm{LiCl}$ & 0,1637 & 21399 \\
\hline & $\mathrm{Li}_{2} \mathrm{SO}_{4}$ & 0,1262 & 10119 \\
\hline \multirow[t]{2}{*}{$\mathrm{Li}_{2} \mathrm{O}$} & $\mathrm{LiCl}$ & 0,3524 & 54698 \\
\hline & $\mathrm{Li}_{2} \mathrm{SO}_{4}$ & 0,2718 & 43418 \\
\hline \multirow[t]{2}{*}{$\mathrm{Mg}$} & $\mathrm{MgO}$ & 0,6032 & 78044 \\
\hline & $\mathrm{Mg}_{2} \mathrm{P}_{2} \mathrm{O}_{7}$ & 0,2184 & 33923 \\
\hline $\mathrm{MgO}$ & $\mathrm{Mg}_{2} \mathrm{P}_{2} \mathrm{O}_{7}$ & 0,3621 & 55879 \\
\hline \multirow[t]{4}{*}{$\mathrm{Mn}$} & $\mathrm{Mn}_{3} \mathrm{O}_{4}$ & 0,7203 & $85 \% 49$ \\
\hline & $\mathrm{Mn}_{2} \mathrm{P}_{2} \mathrm{O}_{7}$ & 0,3869 & 58761 \\
\hline & $\mathrm{MnS}$ & 0,6314 & 80029 \\
\hline & $\mathrm{MnSO}_{4}$ & 0,3638 & 56083 \\
\hline \multirow[t]{4}{*}{$\mathrm{MnO}$} & $\mathrm{Mn}_{3} \mathrm{O}_{4}$ & 0,9301 & 96851 \\
\hline & $\mathrm{Mn}_{2} \mathrm{P}_{2} \mathrm{O}_{7}$ & 0,4998 & 69876 \\
\hline & MnS & 0,8153 & 91131 \\
\hline & $\mathrm{MnSO}_{4}$ & 0,4697 & 67185 \\
\hline Mo & $\mathrm{MoO}_{3}$ & 0,6667 & 82391 \\
\hline \multirow[t]{3}{*}{$\mathrm{N}$} & $\mathrm{NH}_{4} \mathrm{Cl}$ & 0,2619 & 41803 \\
\hline & $\left(\mathrm{NH}_{4}\right)_{2} \mathrm{PtCl}_{6}$ & 0,0631 & 79999 \\
\hline & $\mathrm{Pt}$ & 0,1436 & 15693 \\
\hline \multirow[t]{3}{*}{$\mathrm{NH}_{3}$} & $\mathrm{NH}_{4} \mathrm{Cl}$ & 0,3184 & 50229 \\
\hline & $\left(\mathrm{NH}_{4}\right)_{2} \mathrm{PtCl}_{6}$ & 0,0767 & 88488 \\
\hline & $\mathrm{Pt}$ & 0,1745 & 24182 \\
\hline \multirow{3}{*}{$\mathrm{NH}_{4}$} & $\mathrm{NH}_{4} \mathrm{Cl}$ & 0,3372 & 52789 \\
\hline & $\left(\mathrm{NH}_{4}\right)_{2} \mathrm{PtCl}_{6}$ & 0,0813 & 90985 \\
\hline & $\mathrm{Pt}$ & 0,1848 & 26679 \\
\hline \multirow[t]{5}{*}{$\mathrm{NO}_{3}$} & $\mathrm{C}_{20} \mathrm{H}_{16} \mathrm{~N}_{4} \cdot \mathrm{HNO}_{3}$ & 0,1653 & 21821 \\
\hline & $\mathrm{NH}_{4} \mathrm{Cl}$ & 1,1591 & 06410 \\
\hline & $\left(\mathrm{NH}_{4}\right)_{2} \mathrm{PtCl}_{6}$ & 0,2793 & 44606 \\
\hline & $\mathrm{NO}$ & 2,0664 & 31521 \\
\hline & $\mathrm{Pt}$ & 0,6353 & 80300 \\
\hline \multirow[t]{5}{*}{$\mathrm{N}_{2} \mathrm{O}_{5}$} & $\mathrm{C}_{20} \mathrm{H}_{16} \mathrm{~N}_{4} \cdot \mathrm{HNO}_{3}$ & 0,1440 & 15822 \\
\hline & $\mathrm{NH}_{4} \mathrm{Cl}$ & 1,0095 & 00412 \\
\hline & $\left(\mathrm{NH}_{4}\right)_{2} \mathrm{PtCl}_{6}$ & 0,2432 & 38608 \\
\hline & NO & 1,7998 & 25522 \\
\hline & $\mathrm{Pt}$ & 0,5534 & 74302 \\
\hline $\mathrm{Na}$ & $\mathrm{NaCl}$ & 0,3934 & 59487 \\
\hline
\end{tabular}




\begin{tabular}{|c|c|c|c|}
\hline $\begin{array}{l}\text { Gesucht } \\
\text { cherché }\end{array}$ & $\begin{array}{l}\text { Gefunden } \\
\text { trouvé }\end{array}$ & $\begin{array}{l}\text { Faktor } \\
\text { facteur }\end{array}$ & $\begin{array}{l}\text { Log. (Mantissen) } \\
\text { log. (mantisses) }\end{array}$ \\
\hline \multirow{3}{*}{$\begin{array}{l}\mathrm{Na} \\
\mathrm{Na}_{2} \mathrm{O}\end{array}$} & $\mathrm{Na}_{2} \mathrm{SO}_{4}$ & 0,3238 & .51025 \\
\hline & $\mathrm{NaCl}$ & 0,5303 & $\because \because 450$ \\
\hline & $\mathrm{Na}_{2} \mathrm{SO}_{4}$ & 0,4364 & (i.3989 \\
\hline \multirow[t]{2}{*}{$\mathrm{Ni}$} & $\mathrm{NiO}$ & 0,7858 & $895: 9$ \\
\hline & $\mathrm{NiC}_{8} \mathrm{~N}_{14} \mathrm{~N}_{4} \mathrm{O}_{4}$ & 0,2032 & 30784 \\
\hline \multirow[t]{2}{*}{$\mathrm{NiO}$} & $\mathrm{Ni}$ & 1,2727 & 10471 \\
\hline & $\mathrm{NiC}_{8} \mathrm{H}_{14} \mathrm{~N}_{4} \mathrm{O}_{4}$ & 0,2586 & $+125 \%$ \\
\hline \multirow[t]{3}{*}{$\mathrm{P}$} & $\mathrm{Mg}_{2} \mathrm{P}_{2} \mathrm{O}_{7}$ & 0,2787 & 44579 \\
\hline & $*\left(\mathrm{NH}_{4}\right)_{3} \mathrm{PO}_{4} \cdot 12 \mathrm{MoO}_{3}$ & 0,0164 & $\because 1431$ \\
\hline & $\mathrm{P}_{2} \mathrm{O}_{5} \cdot 24 \mathrm{MoO}_{3}$ & 0,0173 & 23689 \\
\hline \multirow[t]{3}{*}{$\mathrm{P}_{2} \mathrm{O}_{5}$} & $\mathrm{Mg}_{2} \mathrm{P}_{2} \mathrm{O}_{7}$ & 0,6379 & $804 \%$ \\
\hline & $*\left(\mathrm{NH}_{4}\right)_{3} \mathrm{PO}_{4} \cdot 12 \mathrm{MoO}_{3}$ & 0,0375 & 274.38 \\
\hline & $\mathrm{P}_{2} \mathrm{O}_{5} \cdot 24 \mathrm{MoO}_{3}$ & 0,0395 & 59648 \\
\hline \multirow{3}{*}{$\mathrm{P}()_{4}$} & $\mathrm{Mg}_{2} \mathrm{P}_{2} \mathrm{O}_{7}$ & $0,858.5$ & $9: 3118$ \\
\hline & $*\left(\mathrm{NH}_{4}\right)_{3} \mathrm{PO}_{4} \cdot 12 \mathrm{MoO}_{3}$ & $0,0,502$ & 80079 \\
\hline & $\mathrm{P}_{2} \mathrm{O}_{5} \cdot 12 \mathrm{MoO}_{3}$ & 0,0528 & 20288 \\
\hline \multirow[t]{5}{*}{$\mathrm{Pb}$} & $\mathrm{PbCrO}_{4}$ & 0,6411 & $8069:$ \\
\hline & $\mathrm{PbO}$ & 0,9283 & $967 \% 0$ \\
\hline & $\mathrm{PbO}_{2}$ & 0,8662 & 93763 \\
\hline & $\mathrm{PbS}$ & 0,8660 & $93 \% 50$ \\
\hline & $\mathrm{PbSO}_{4}$ & $0,68: 31$ & 83456 \\
\hline \multirow[t]{4}{*}{$\mathrm{Pl}, \mathrm{O}$} & $\mathrm{PbCrO}_{4}$ & 0,6906 & $8392 \%$ \\
\hline & $\mathrm{PbO}_{2}$ & 0,9381 & 96999 \\
\hline & PbS & 0,9328 & 96.980 \\
\hline & $\mathrm{PbSO}_{4}$ & 0,7360 & $86 j 686$ \\
\hline $\mathrm{Pbs}$ & $\mathrm{PbSO}_{4}$ & 0,7890 & 89706 \\
\hline$R b$ & $\mathrm{Rb}_{2} \mathrm{SO}_{4}$ & 0,64083 & 80631 \\
\hline $\mathrm{S}$ & $\mathrm{BaSO}_{4}$ & 0,1374 & 13787 \\
\hline$S O)_{2}$ & $\mathrm{BaSO}_{4}$ & (1),2744 & 4.3842 \\
\hline $\mathrm{SO}_{3}$ & $\mathrm{BaSO}_{4}$ & 0,3430 & 5.3504 \\
\hline$S()_{4}$ & $\mathrm{BaSO}_{4}$ & 0,4115 & ij $436 i$ \\
\hline $\mathrm{SO})_{4} \mathrm{H}_{2}$ & $\mathrm{BaSO}_{4}$ & 0,4201 & $6: 338$ \\
\hline $\mathrm{Sl}$ & $\mathrm{Sb}_{2} \mathrm{O}_{4}$ & 0,7898 & 89749 \\
\hline & $\mathrm{Sb}_{2} \mathrm{~S}_{3}$ & $0,71+2$ & 8.5382 \\
\hline & $\mathrm{Sb}_{2} \mathrm{~S}_{5}$ & 0,5998 & 7800 \\
\hline
\end{tabular}

*) empirisch. 


\begin{tabular}{|c|c|c|c|}
\hline $\begin{array}{l}\text { G'esucht } \\
\text { cherché }\end{array}$ & $\begin{array}{l}\text { Gefunden } \\
\text { trouvé }\end{array}$ & $\begin{array}{l}\text { Faktor } \\
\text { facteur }\end{array}$ & $\begin{array}{l}\text { Log. (Mantissen) } \\
\text { log. (mantisses) }\end{array}$ \\
\hline $\mathrm{Sb}_{2} \mathrm{O}_{3}$ & $\begin{array}{l}\mathrm{Sb}_{2} \mathrm{O}_{4} \\
\mathrm{Sb}_{2} \mathrm{~S}_{3} \\
\mathrm{Sb}_{2} \mathrm{~S}_{5}\end{array}$ & $\begin{array}{l}0,9475 \\
0,8568 \\
0,7196 \\
\end{array}$ & $\begin{array}{l}97656 \\
93289 \\
85707\end{array}$ \\
\hline $\begin{array}{l}\mathrm{SeO}_{2} \\
\mathrm{SeO}_{3}\end{array}$ & $\begin{array}{l}\mathrm{Se} \\
\mathrm{Se}\end{array}$ & $\begin{array}{l}1,4040 \\
1,6061\end{array}$ & $\begin{array}{l}14737 \\
20576 \\
\end{array}$ \\
\hline $\begin{array}{l}\mathrm{Si} \\
\mathrm{SiO}_{3} \\
\mathrm{SiO}_{4}\end{array}$ & $\begin{array}{l}\mathrm{SiO}_{2} \\
\mathrm{SiO}_{2} \\
\mathrm{SiO}_{2}\end{array}$ & $\begin{array}{l}0,4693 \\
1,2653 \\
1,5307\end{array}$ & $\begin{array}{l}67147 \\
10221 \\
18488\end{array}$ \\
\hline $\begin{array}{l}\mathrm{Sn} \\
\mathrm{SnO}_{2}\end{array}$ & $\begin{array}{l}\mathrm{SnO}_{2} \\
\mathrm{Sn}\end{array}$ & $\begin{array}{l}1,7877 \\
1,2696\end{array}$ & $\begin{array}{l}89634 \\
10366\end{array}$ \\
\hline $\mathrm{SrO}$ & $\begin{array}{l}\mathrm{SrCO}_{3} \\
\mathrm{SrSO}_{4} \\
\mathrm{Sr}\left(\mathrm{NO}_{3}\right)_{2} \\
\mathrm{SrCO}_{3} \\
\mathrm{SrSO}_{4} \\
\mathrm{Sr}\left(\mathrm{NO}_{3}\right)_{2}\end{array}$ & $\begin{array}{l}0,5935 \\
0,4769 \\
0,4140 \\
0,7019 \\
0,5641 \\
0,4896\end{array}$ & $\begin{array}{l}77341 \\
67846 \\
61701 \\
84627 \\
75132 \\
68980\end{array}$ \\
\hline $\begin{array}{l}\mathrm{TeO}_{2} \\
\mathrm{TeO}_{3}\end{array}$ & $\begin{array}{l}\mathrm{Te} \\
\mathrm{Te}\end{array}$ & $\begin{array}{l}1,2510 \\
1,3765\end{array}$ & $\begin{array}{l}09 \% 25 \\
1387 \%\end{array}$ \\
\hline Th & $\begin{array}{l}\mathrm{Th}\left(\mathrm{NO}_{3}\right)_{4} \cdot 4 \mathrm{H}_{2} \mathrm{O} \\
\mathrm{ThO}_{2}\end{array}$ & $\begin{array}{l}0,4207 \\
0,8790\end{array}$ & $\begin{array}{l}62392 \\
94398\end{array}$ \\
\hline $\mathrm{Ti}$ & $\mathrm{TiO}_{2}$ & 0,6005 & $7 \% 851$ \\
\hline $\mathrm{U}$ & $\begin{array}{l}\mathrm{U}_{3} \mathrm{O}_{8} \\
\mathrm{UO}_{2}\end{array}$ & $\begin{array}{l}0,8481 \\
0,8816 \\
\end{array}$ & $\begin{array}{l}92844 \\
94525\end{array}$ \\
\hline $\mathrm{V}$ & $\mathrm{V}_{2} \mathrm{O}_{5}$ & 0,5614 & 74928 \\
\hline$W$ & $\mathrm{WO}_{3}$ & 0,7931 & 89933 \\
\hline $\mathrm{Y}$ & $\mathrm{Y}_{2} \mathrm{O}_{3}$ & 0,7871 & 89600 \\
\hline $\mathrm{ZnO}$ & $\begin{array}{l}\mathrm{ZnO} \\
\mathrm{ZnS} \\
\mathrm{ZnNH}_{4} \mathrm{PO}_{4} \\
\mathrm{Zn}_{2} \mathrm{P}_{2} \mathrm{O}_{7} \\
\mathrm{ZnS} \\
\mathrm{ZnNH}_{4} \mathrm{PO}_{4} \\
\mathrm{Zn}_{2} \mathrm{P}_{2} \mathrm{O}_{7}\end{array}$ & $\begin{array}{l}0,8034 \\
0,6709 \\
0,3664 \\
0,4290 \\
0,8351 \\
0,4561 \\
0,5340 \\
\end{array}$ & $\begin{array}{l}90491 \\
82664 \\
56395 \\
63248 \\
92173 \\
65904 \\
72756 \\
\end{array}$ \\
\hline $\mathrm{Zr}$ & $\mathrm{ZrO}$ & 0,7390 & 86864 \\
\hline
\end{tabular}

